\title{
GRAGOATÁ:
}

Revista dos Programas de Pós-Cutuat oúto do Instituto de Letras

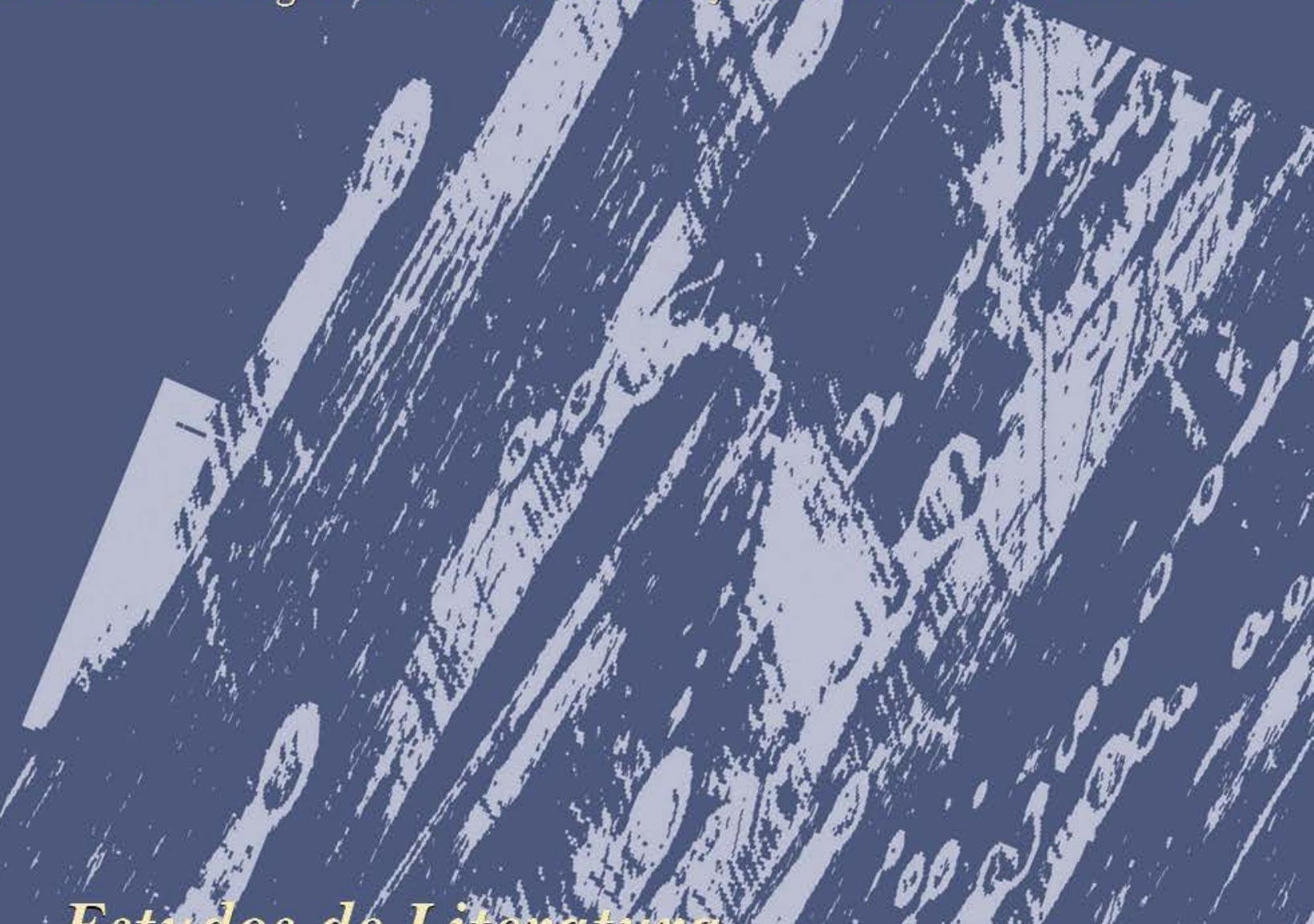

Estudos de Literch

it








\section{Política Editorial}

A Revista Gragoatá tem como objetivo a divulgação nacional e internacional de ensaios inéditos, de traduções de ensaios e resenhas de obras que representem contribuições relevantes tanto para reflexão teórica mais ampla quanto para a análise de questões, procedimentos e métodos específicos nas áreas de Língua e Literatura.

eISSN 2358-4114

v. 22

n. 43

p. $566-959$ mai.-ago. 2017 
Direitos desta edição reservados aos Programas de Pós-Graduação do Instituto de Letras da Universidade Federal Fluminense

Rua Professor Marcos Waldemar de Freitas Reis, s/nº. Campus do Gragoatá, Bloco C, sala 518. CEP: 24210-201, Niterói / RJ.

É proibida a reprodução total ou parcial desta obra sem autorização expressa dos editores.

Editoras:

Editora Assistente:

Organização:

Projeto gráfico:

Capa:

Revisão (português):

Revisão (inglês):

Assessoria Executiva:

Diagramação:

Periodicidade:
Lucia Teixeira (Estudos de Linguagem) e Paula Glenadel (Estudos de Literatura)

Silvia Sousa (Estudos de Linguagem) e Viviana Gelado (Estudos de Literatura)

André Dias e Kenneth David Jackson

Estilo \& Design Editoração Eletrônica Ltda. ME

Rogério Martins

Daniel Felix Costa Júnior, Eduardo Castro, Gilson Magalhães de Carvalho, Ivana Quintão de Andrade, Ricardo Augusto Gonçalves e Rita Isadora Pessoa

\section{Eduardo Castro e Rita Isadora Pessoa}

Elir Ferrari

Mauro Corrêa Filho

Quadrimestral
Esta edição contou com o apoio da

(c) FAPERJ

Dados Internacionais de Catalogação na Publicação

G737 Gragoatá / Programas de Pós-Graduação do Instituto de Letras da Universidade Federal Fluminense.- n. 1 (1996) - . - Niterói : UFF, 1996.

v. il.

Semestral - 1996-2016

Quadrimestral - 2017-

A partir do número 36 de 2014, a publicação passa a ser somente online. ISSN 2358-4114 (online) - ISSN 1413-9073 (impresso, até o no 35/2013)

1. Literatura. 2. Linguística. I. Universidade Federal Fluminense. Programa de Pós-Graduação em Letras.

CDD 800 


\title{
APOIO PROPPI / UNIVERSIDADE FEDERAL FLUMINENSE
}

\author{
Reitor: Sidney Luiz de Matos Mello \\ Vice-Reitor: Antonio Claudio Lucas da Nóbrega \\ Pró-Reitor de Pesquisa e Pós-Graduação e Inovação: \\ Diretora do Instituto de Letras: \\ Vice-diretora do Instituto de Letras: \\ Coordenadora da Pós-Graduação em Estudos de Linguagem: \\ Vice-coordenadora da Pós-Graduação em Estudos de Linguagem: \\ Coordenador da Pós-Graduação em Estudos de Literatura: \\ Vice-coordenadora da Pós-Graduação em Estudos de Literatura: \\ Vitor Francisco Ferreira \\ Ida Maria Santos Ferreira Alves \\ Telma Cristina de Almeida Silva Pereira \\ Mônica Maria Guimarães Savedra \\ Silmara Cristina Dela da Silva \\ José Luís Jobim de Salles Fonseca \\ Renata Flavia da Silva
}

Editores Associados: André Dias, UFF

Eduardo Kenedy, UFF

Jacques Fontanille, Université de Limoges, França José Luís Jobim, UFF

Kenneth David Jackson, Yale University, EUA

Luís Maffei, UFF

Mônica Guimarães Savedra, UFF

Silvia Sousa, UFF

Vanise Gomes Medeiros, UFF

Viviana Gelado, UFF

Comissão Editorial: Alessandro Zinna - Université de Toulouse, França Ana Pizarro - Universidad de Santiago, Chile Arnaldo Cortina - UNESP-Araraquara

Augusto Soares da Silva - Universidade Católica Portuguesa, Portugal

Bethania Sampaio Correia Mariani - UFF

Célia Pedrosa - UFF

Cláudia Poncioni - Université de Paris III - Sorbonne Nouvelle, França

Cleonice Berardinelli - UFRJ

Dermeval da Hora - UFPB

Eneida Leal Cunha - UFBA/PUC-Rio

Eneida Maria de Souza - UFMG

Erotilde Goreti Pezatti - UNESP-SJRP

Eurídice Figueiredo - UFF

Evanildo Bechara - UER

Freda Indursky - UFRGS

Hélder Macedo - King's College, Inglaterra

Henrique Monteagudo - Universidad de Santiago de Compostela, Espanha

Jacqueline Authier-Revuz - Université Paris III, França Jacqueline Penjon - Université de Paris III - Sorbonne Nouvelle, França

Jean Cristtus Portela - Universidade Estadual Paulista-Araraquara, Brasil

Jean-Marie Fournier - Université de Paris III - Sorbonne Nouvelle, França

José Carlos de Azeredo - UERJ

José Luiz Fiorin - USP

Kathrin Sartingen - Universität Wien, Áustria

Konstanze Jungbluth - Europa-Universität Viadrina Frankfurt (Oder), Alemanha

Laura Padilha - UFF

Leila Bárbara - PUC-SP

Lourenço de Rosário - Fundo Bibliográfico de Língua Portuguesa, Portugal

Luiz Amaral - University of Massachusetts, EUA

Luiz Francisco Dias - UFMG
Mabel Moraña - Saint Louis University, EUA

Malcolm Coulthard - University of Birmingham, Inglaterra

Márcia Maria Valle Arbex - UFMG

Márcia Paraquet - UFBA

Marcos Antonio Siscar - UNICAMP

Marcus Maia - UFRJ

Margarida Calafate Ribeiro - Universidade de Coimbra, Portugal

Maria Angélica Furtado da Cunha - UFRN

Maria Eugênia Lamoglia Duarte - UFRJ

Maria Luiza Braga - UFRJ

Mariangela Rios de Oliveira - UFF

Marie-Anne Paveau - Université de Paris XIII - Sorbonne Nouvelle, França

Marlene Correia - UFRJ

Mieke Bal - Universiteit van Amsterdam, Holanda

Nádia Battela Gotlib - USP

Nélson H. Vieira - Brown University, EUA

Olinda Kleiman - Université de Paris III - Sorbonne Nouvelle, França

Regina Zilberman - UFRGS

Roberto G. de Almeida - Concordia University, Canadá

Roberto Vecchi - Università degli Studi di Bologna, Itália

Roger Chartier - Collège de France, França

Rosa Maria Martelo - Universidade do Porto, Portugal

Ria Lemaire - Université de Poitiers, França

Silviano Santiago - UFF

Silvio Renato Jorge - UFF

Sírio Possenti - UNICAMP

Solange Vereza - UFF

Teun van Dijk - Universitat Pompeo Fabra, Barcelona, Espanha

Vera Menezes - UFMG

Vilma Arêas - UNICAMP

Walter Moser - Université de Montréal, Canadá 